\title{
Longitudinal diffusion tensor imaging shows progressive changes in white matter in Huntington's disease
}

Sarah Gregory ${ }^{\mathrm{a}}$, James H. Cole ${ }^{\mathrm{b}, \mathrm{c}^{*}}$, Ruth E. Farmer ${ }^{\mathrm{d}}$, Elin M. Rees ${ }^{\mathrm{b}}$, Raymund A.C. Roos ${ }^{\mathrm{e}}$, Reiner

Sprengelmeyer ${ }^{\mathrm{f}}$, Alexandra Durr ${ }^{\mathrm{g}}$, Bernhard Landwehrmeyer ${ }^{\mathrm{f}}$, Hui Zhang ${ }^{\mathrm{h}}$, Rachael I. Scahill ${ }^{\mathrm{b}}$, Sarah J.

Tabrizi $^{b}$, Chris Frost ${ }^{d}$, Nicola Z. Hobbs ${ }^{b, i}$

*Equal first author

a Wellcome Trust Centre for Neuroimaging, UCL, London, WC1N 3BG, UK

${ }^{\mathrm{b}}$ UCL Institute of Neurology, University College London, UK

'Computational, Cognitive \& Clinical Neuroimaging Laboratory, Department of Medicine, Imperial

College London, UK

${ }^{\mathrm{d}}$ Department of Medical Statistics, London School of Hygiene \& Tropical Medicine

eDepartment of Neurology, Leiden University Medical Centre, 2300RC Leiden, The Netherlands

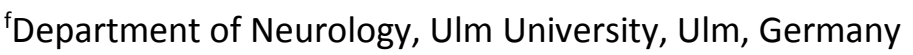

${ }^{\text {g} D e p a r t m e n t ~ o f ~ G e n e t i c s ~ a n d ~ C y t o g e n e t i c s, ~ I N S E R M ~ U M R ~ S 679, ~ A P H P ~ H o ̂ p i t a l ~ d e ~ l a ~ S a l p e ̂ t r i e ̀ r e, ~ P a r i s, ~}$ France

${ }^{\text {h}}$ Centre for Medical Image Computing, University College London, UK

iIXICO PIC., London, UK

\section{Corresponding author}

Sarah Gregory

Wellcome Trust Centre for Neuroimaging at University College London

12 Queen Square, London, WC1N 3BG

Email: s.gregory@ucl.ac.uk

Telephone: 02034484366

Running Title: Longitudinal DTI in Huntington's disease 


\begin{abstract}
BACKGROUND: Huntington's disease is marked by progressive neuroanatomical changes, assumed to underlie the development of the disease's characteristic symptoms. Previous work has demonstrated longitudinal macrostructural white-matter atrophy, with some evidence of microstructural change focused in the corpus callosum.
\end{abstract}

OBJECTIVE: To more accurately characterise longitudinal patterns, we examined white matter microstructural change using Diffusion Tensor Imaging (DTI) data from three timepoints over a 15 month period.

METHODS: In 48 early-stage HD patients and 36 controls from the multi-site PADDINGTON project, diffusion tensor imaging (DTI) was employed to measure changes in fractional anisotropy (FA) and axial (AD) and radial diffusivity (RD) in 24 white matter regions-of-interest (ROIs).

RESULTS: Cross-sectional analysis indicated widespread baseline group differences, with significantly decreased FA and increased $A D$ and RD found in HD patients across multiple ROIs. Longitudinal rates of change differed between HD patients and controls in the genu and body of corpus callosum, corona radiata and anterior limb of internal capsule. Change in RD in the body of the corpus callosum was associated with baseline disease burden, but other clinical associations were not significant.

CONCLUSIONS: We detected subtle longitudinal white matter changes in early HD patients. Progressive white matter abnormalities in HD may not be uniform throughout the brain, with some areas remaining static in the early symptomatic phase. Longer assessment periods across disease stages will help map this progressive trajectory.

Keywords: Huntington's disease, Diffusion Tensor Imaging, Longitudinal, Symptomatic 


\section{Introduction}

Huntington's disease (HD) causes progressive neurodegeneration, leading to debilitating motor, cognitive and psychiatric symptoms. Despite identification of the gene responsible for HD [1] and subsequent years of concerted clinical and research efforts, the search for disease-modifying treatments is still in its infancy.

Studying HD in vivo using magnetic resonance imaging (MRI) is a powerful approach for improving our understanding of the disease's progressive nature. Used to elucidate aspects of the neural pathogenesis of HD in the striatum, studies using volumetric approaches have also shown that HD pathology extends to macrostructural abnormalities within cortical and white matter regions [2-6]. By employing diffusion tensor imaging (DTI), we can similarly investigate microstructural changes in white matter with several studies already having identified abnormalities in manifest HD genecarriers [7-11], pre-manifest HD (preHD) gene carriers [12-17] and across multiple disease stages [5, 18-30].

Cross-sectional DTI studies which have included both preHD and early-HD gene-carriers have attempted to characterise disease progression, but this is better achieved by conducting longitudinal analyses which can map individual patterns of change. There is strong evidence of progressive macrostructural white matter degeneration [31-39] and current evidence suggests that longitudinal change in caudate nucleus volume is a particularly promising candidate neuroimaging biomarker for future clinical trials [36]. If the microstructural properties captured by DTI are more sensitive to the cellular processes underlying neurodegeneration [40], DTI may potentially be a better index of HD progression than macrostructural volumetric methods. Thus, further longitudinal DTI analysis of white matter in HD is warranted. However, only a limited number of studies have examined DTI in HD longitudinally (see Rees et al., for review [41]) and these have generally either been restricted to striatal grey matter regions $[42,43]$, or limited in sample size and scope. For example, white matter change was investigated in a small combined group $(N=7)$ of early HD and preHD gene carriers 
using whole-brain tract-based spatial statistics (TBSS) [44, 45]. This preliminary research identified longitudinal decreases in fractional anisotropy (FA) and axial diffusivity (AD) after one year in the HD patients when compared to a matched control group. Conversely, Sritharan and colleagues [46] were unable to detect similar changes over the same interval, using a region-of-interest (ROI) approach in a larger sample of 18 HD patients and 17 controls. However, this analysis only examined mean diffusivity (MD) of the corpus callosum. A recent study has investigated longitudinal change over 18 months using data from two timepoints in a larger group of preHD and early HD genecarriers [29]. Again, using TBSS, they have shown that when compared to both preHD and control groups, early HD gene-carriers have reduced FA in the genu, body and splenium of the corpus callosum and mid cingulum and that callosal FA levels are predicted by the Unified Huntington's Disease Rating Scale Total Motor Score (UHDRS-TMS)[47]. Furthermore, there was evidence of increased radial diffusivity (RD) in the corpus callosum, cingulum, internal capsule and striatal projections when compared to the premanifest, but not the control group.

Another recent study applying histogram analysis to DTI data over 24 months using data from two timepoints, assessed microstructural properties of whole-brain gray matter, white matter and the striatum in different stages of HD [30]. In that study evidence was found for alterations in crosssectional diffusion profiles between early manifest HD and preHD subjects compared to healthy controls, without evidence for longitudinal differences in the degree of diffusivity change between the groups. Investigating the structural connectome using the same dataset by applying graph theoretical analysis, Odish and colleagues revealed longitudinal differences between early manifest HD, preHD subjects and controls in various network measures[48]. These results further emphasise the importance of exploring different analytical approached to study the biomarker potential of diffusion MRI.

The aim of the current study was to investigate progressive alterations in white matter microstructure during the early stages of symptomatic HD using multi-site DTI data combined with an ROI approach. To more clearly characterise longitudinal change, we used three timepoints over a 
15 month interval to track the subtle, non-linear changes in progression across this period. We focussed on FA as a measure of the direction of diffusivity, in addition to both $A D$ and RD which respectively index water movement parallel and perpendicular to the main fibre. We investigated white matter changes with respect to genetic factors (i.e. CAG repeat length) and disease burden, as these markers of HD show associations with cross-sectional white matter abnormalities $[8,23,24]$ and in using the ROI approach, this analysis was designed to highlight specific brain regions for future evaluation as DTI-derived biomarkers for potential therapeutic studies.

\section{Materials and Methods}

\section{Participants}

$61 \mathrm{HD}$ patients and 40 control participants were recruited as part of the PADDINGTON study [11] across four study sites (Leiden, Netherlands; London, UK; Paris, France; Ulm, Germany). Participants attended three separate study visits, with an initial baseline and subsequent 6-month and 15-month follow-ups. Due to a combination of participant dropout, missing data and quality control failure, 17 participants (13 HD patients, 4 controls) did not have complete diffusion MRI datasets for all three visits (two missing 6-month assessment; 15 missing 15-month); hence 84 participants (48 HD patients and 36 controls - see Table 1) were included in the final analysis. These exclusions were necessary as the longitudinal image registration scheme outlined below cannot account for missing image data. Excluded HD patients had marginally higher disease burden and Total Motor Score (TMS) and lower Total Functional Capacity (TFC) at baseline but overall the demographic differences were minimal (see Supplementary Table 1).

In addition to MRI data, behavioural, cognitive and clinical assessments were performed at each visit. All patients had received a diagnosis of manifest HD, were at disease stage I [49] and had a baseline UHDRS TFC score between 11-13. One patient had a TFC score of 9 and was classified as disease stage II (this score remained stable across all study visits). CAG repeat length was assessed to confirm diagnosis and all patients had between 39-54 repeats. Control participants were spouses, 
partners or gene-negative siblings of the HD patients. Inclusion criteria were: $18-65$ years of age, no diagnosis of psychiatric or other neurological disorders, not currently taking part in other pharmacological research and no contraindication to MRI scanning. The respective local ethical committees for each study site approved the study and written informed consent was obtained from each participant.

\section{Data acquisition}

3T MRI Diffusion-weighted T1 structural and DTI images were acquired at all sites.

T1 Imaging parameters: For the Siemens Tim Trio and Verio scanners (London and Paris respectively) the T1 MP-RAGE scans were acquired with the following parameters: $T R=2200 \mathrm{~ms}, \mathrm{TE}=2.2 \mathrm{~ms}$, flip angle $=10^{\circ}, \mathrm{FOV}=28 \mathrm{~cm}$, matrix size $=256 \times 256$, yielding 208 sagittal slices with a slice thickness of $1.0 \mathrm{~mm}$ with no inter-slice gap. For the Siemens Allegra (Ulm), parameters were as above except TE $=2.81 \mathrm{~ms}$, flip angle $=9^{\circ}$ and a slice thickness of $1.1 \mathrm{~mm}$ For the Philips Achieva scanner (Leiden) T1weighted magnetisation-prepared rapid gradient echo (MP-RAGE) scans were acquired with the following parameters; $T R=7.7 \mathrm{~ms}, \mathrm{TE}=3.5 \mathrm{~ms}$, flip angle $=8^{\circ}, \mathrm{FOV}=24 \mathrm{~cm}$, matrix size $=224 \times 224$, yielding 164 sagittal slices to cover the entire brain with a slice thickness of $1.0 \mathrm{~mm}$ with no interslice gap.

DTI Imaging Parameters: For the Siemens Tim Trio (London) DTI data were acquired using an EPI sequence with the following parameters: 65 axial slices of $2 \mathrm{~mm}$ thickness, with no inter-slice gaps, acquisition matrix $=96 \times 128$, in-plane resolution of $2 \mathrm{~mm}^{2}$, resulting in isotropic voxels $(T R=7600$ $\mathrm{ms}, \mathrm{TE}=84 \mathrm{~ms}$ ). For the Siemens Verio (Paris) DTI data were acquired using an EPI sequence with the following parameters; 75 axial slices of $2 \mathrm{~mm}$ thickness, with no inter-slice gaps, acquisition matrix $=128 \times 128$, in-plane resolution of $2 \mathrm{~mm}^{2}$, resulting in isotropic voxels $(T R=13100 \mathrm{~ms}, \mathrm{TE}=86$ $\mathrm{ms}$ ). For both, data were acquired in 42 different encoding directions with $\mathrm{b}=1000 \mathrm{~s} / \mathrm{mm}^{2}$, along with $7 \mathrm{~b}=0$ images. For the Siemens Allegra $(\mathrm{U} / \mathrm{m})$, each data volume consisted of 52 axial slices of $2.2 \mathrm{~mm}$ thickness, with no inter-slice gaps, acquisition matrix $=96 \times 128$, in-plane resolution of 2.2 
$\mathrm{mm}^{2}$, resulting in isotropic voxels $(\mathrm{TR}=7600 \mathrm{~ms}, \mathrm{TE}=85 \mathrm{~ms})$. Diffusion data were acquired in 47 different encoding directions with $b=1000 \mathrm{~s} / \mathrm{mm}^{2}$, along with three $b=0$ images. For the Philips Achieva scanner (Leiden) DTI data were acquired using the following parameters; 55 axial slices of 2 $\mathrm{mm}$ thickness, with no inter-slice gaps, acquisition matrix $=112 \times 112$, in-plane resolution of $2 \mathrm{~mm}^{2}$, resulting in isotropic voxels (TR $=8062 \mathrm{~ms}, \mathrm{TE}=56 \mathrm{~ms}$ ). Diffusion data were acquired in 42 different encoding directions with $b=1000 \mathrm{~s} / \mathrm{mm}^{2}$, along with one $b=0$ image.

For further details on acquisition parameters and pooling of multi-site site data see our previous work on this sample $[11,50]$. Visual quality control was performed to check for acquisition protocol compliance, artefacts and head positioning. Rescans were requested for four participants and scans were excluded due to failure to meet the required quality criteria, leaving 84 participants for the analysis. All image processing and analysis was performed blinded to participant diagnosis. All site effects were modelled within the analysis.

\section{Data pre-processing}

Diffusion-weighted images were initially registered to the corresponding b0 reference image to correct for motion and eddy current distortions, with updates applied to the gradient scheme accordingly. Subsequently, a non-linear least-squares method was used to fit the tensor at each voxel, using Camino [51]. Tensor-based registration was then performed using DTI-TK (http://dtitk.sourceforge.net) [52]. This offers improvements over conventional scalar-based registration as it incorporates information from local fibre orientations and has been shown to improve the sensitivity of DTI metrics to microstructural group differences $[53,54]$ and the performance of study-specific atlas generation [55]. We employed a well-established specific longitudinal registration procedure, which registers average within-subject templates to a study-specific group template; this avoids biases caused by asymmetric image registration or imbalanced application of interpolation schemes [56] (see Figure 1). In short, for every participant, tensor images for each visit were co-registered together to form a 'bootstrap' template, then each image was non-linearly registered to the 
template using an iterative approach. The resulting affine and non-linear registrations were then combined so that the input images could be mapped to subject-template space in one interpolation step [57]. A group template was then created using this same iterative affine and non-linear procedure; with participant average images as input. Again, transformations were combined to take within-participant template images to group template space with one interpolation. This resulted in all participants' tensor images being spatially normalised to a study-specific group template, from which individual subject maps for $F A, A D$ and $R D$ could be generated for further processing and statistical analysis.

\section{Atlas-based ROI analysis}

To define our ROls, we used the $2 \mathrm{~mm}^{3}$ ICBM-DTI-81 atlas [58] employed within FSL software. Based on our previous work [59], the number of regions was reduced from 48 to 24 to include only those with high test-retest reliability for FA, AD and RD (i.e. intraclass correlation coefficient $>0.8$ ) (Tables 2 and 3). It should be noted that the current sample includes the subsample reported in [59]. However, the analysis in that study used sequentially acquired data from a single visit, not multiple timepoints, and therefore presents no confound when using that data to inform the selection of ROIs for the present study. The DTI-81 atlas was registered to the group FA template using the NiftyToolkit (http://sourceforge.net/projects/niftyreg) components Aladin, for affine registration [60] and F3D, for non-linear refinement [61]. The computed transformation was then used to warp the label files to match the group FA template. The group FA template was thresholded at FA $>0.2$ and belowthreshold voxels removed from DTI-81 label images in group-template space. The mean value of each metric (FA, AD and RD) was then calculated across each ROI for each participant at each visit.

\section{Statistical analysis}

All analysis was conducted in STATA version 13 . The repeated measures of each $\mathrm{ROI} / \mathrm{metric}$ combination were separately analysed using generalised least squares regression models, with outcome variance (and correlations between pairs of measures) allowed to differ both by disease 
group and by visit. The models included a group factor (HD patient or Control), time from baseline (in days) and a quadratic term that modelled non-linear change over the three visits. Also, baseline covariates of age, sex and study site were included. Interaction terms between time (both linear and quadratic) and all other variables were included. The primary measures of interest were group differences at baseline and in change over 15 months. Due to the large number of ROIs and ensuing increased risk of false positives, a correction for multiple testing was carried out using a procedure to control the False Discovery Rate (FDR) [62] at 5\%. It was considered appropriate to do this for each metric separately, thus corrections were based on analysing 24 ROIs. Due to a priori concern of between-site differences, two interaction terms were tested using the same multiple comparison procedure as above: baseline interaction between group and study site, three-way site by group by time (both linear and quadratic) interaction. If the three-way interaction was significant, then both the baseline group differences and the between-group difference in 15-month change were reported by site. If only the baseline interaction between site and group was significant, this was retained in the model and a single between-group difference in 15-month change reported (nonsignificant three-way interactions were removed for clarity). If neither interaction was statistically significant they were both removed from the model. FDR correction for the primary measures of interest was conducted after removal of any measures that needed to be reported as site-specific.

\section{Clinical associations}

Longitudinal imaging measures were tested for associations with baseline CAG and disease burden score DBS) in the HD participant group only. This was restricted to those measures identified as having shown significant between-group differences in 15-month change. For simplicity, a linear regression was used to model 15-month change with an adjustment for the baseline diffusion value. Additional adjustments were also made for age, sex and study site. Again, interaction between group and site was allowed for, and if significant, site-specific associations were presented. If not, the interaction was removed from the model. As above, $p$-values were adjusted using FDR 
correction at $5 \%$, this time based on the number of measures carried through to the secondary analysis.

\section{Results}

Baseline comparison of early HD patients and controls

To provide context for understanding longitudinal changes, we investigated cross-sectional differences between early HD patients and controls at baseline. All 24 regions showed statistically significant group differences (corrected for multiple comparisons) for at least one metric, with the HD group demonstrating either decreases in FA or increases in $A D$ or RD when compared with controls (Table 2). Regions where all three DTI metrics showed significant differences were the three subdivisions of the corpus callosum (genu, body and splenium), the left external capsule and left bilateral sagittal stratum. There were no regions where increased FA was evident in the HD group, nor where decreased AD or RD was found.

\section{Comparison of longitudinal changes in early HD patients and controls}

DTI metrics showed significant differences in rates of change between early HD patients and controls in nine regions (see Figure 2 and Table 3). The genu and body of the corpus callosum showed reduced FA and increased RD, with increased $A D$ in the genu only. There was increased RD and $A D$ in the left anterior corona radiata; and increases in AD were also evident in both the right superior corona radiate and anterior limb of internal capsule. All differences were significant following multiple correction. Four metrics showed evidence of interaction between group differences in 15month change and site (see Supplementary Table 2).

If between-group differences were driven by disease, we would expect to see a relatively flat trajectory of DTI measures over time for the control group, coupled with a deterioration in HD subjects to reflect disease progression. As an informal check, we plotted the model-estimated trajectories of diffusivity measures throughout the study period in controls and HD groups 
separately. Fitted values are shown for hypothetical participants age 50, with a 50/50 male-female split and a $25 \%$ contribution from each site (Figure 3). Examination of the example trajectories highlighted FA in the genu of corpus callosum, AD in the anterior limb of internal capsule and right superior corona radiata and $\mathrm{RD}$ in the left anterior corona radiata as matching this pattern. The longitudinal trajectory in other regions indicated a possible regression to the mean in controls influencing the detected group differences in rates of change over time.

\section{Clinical associations with longitudinal neuroimaging measures}

The nine measures that reached statistical significance for longitudinal change (Table 3) were examined for associations between 15-month change and baseline CAG and disease burden in the HD subjects. Estimates and 95\% confidence intervals are displayed in Table 4. After correction for multiple comparisons, only RD in the body of the corpus callosum showed a significant association with disease burden, with other measures showing directionally consistent but only borderline statistically significant results. After adjustment for a FDR of $5 \%$ there was no evidence that the associations differed between study sites.

\section{Discussion}

Early-stage HD gene-carriers show progressive changes in white matter microstructure in a number of key tracts when compared with healthy, age-matched controls. Using multi-site data, we have demonstrated widespread cross-sectional differences in diffusivity at baseline between controls and early stage HD, with longitudinal change in a number of key tracts including the genu and body of corpus callosum, left anterior corona radiata, right superior corona radiata and right anterior limb of internal capsule.

Our cross-sectional findings at baseline concur with previous work using the current cohort [11] and much of the previous DTI research examining white matter in early-stage HD, which demonstrated abnormalities in the corpus callosum $[18,19,24,27,63]$ and more widely throughout the brain $[5,7-$ 
$10,20-23,26,28,30]$. We have also shown longitudinal change in the corpus callosum, corona radiate, and right anterior internal capsule across a series of DTI metrics, which supports evidence from previous longitudinal studies $[29,45]$. Weaver et al., for example, identified FA decreases at follow-up in the corpus callosum, internal capsule and corona radiate. Most significantly, however, the recent longitudinal study by Poudel et al. demonstrated that the most robust white matter changes in manifest HD occur within the corpus callosum [29]. Both the current study and that of Poudel have shown that in early HD there are FA increases and RD decreases in the corpus callosum when compared with controls. Although this previous study used only two timepoints, incorporating a longer period of change, and a whole-brain rather than an a priori hypothesis driven method, findings are still highly consistent regarding the corpus callosum. These three studies highlight a consistent disturbance within the corpus callosum above all other white matter tracts. This is compatible with the aforementioned extant evidence at the cross-sectional level which robustly demonstrates white matter abnormalities within the corpus callosum. Despite concordance concerning measures of RD and FA, there is far greater inconsistency in terms of $A D$ measures. We saw increased $A D$ not only in the corpus callosum, but also the anterior and superior corona radiate and the anterior internal capsule. Weaver, however, reported decreased AD in a small set of voxels [45], these differences likely due to low statistical power and variability in terms of disease progression; while Poudel et al. did not show any significant differences in AD at all [29]. Crosssectional studies of early HD patients have previously shown increases in AD compared with controls $[7,11,18,27,30]$ and there is evidence that the negative correlation between $A D$ and $R D$ acts as an independent predictor of HD disease progression (see unpublished data - Mike O). However, given the limited number of studies examining longitudinal change in white matter, further replication of our findings would be required to confirm the increases in $A D$ in early $H D$ gene-carriers. It is important to note that some longitudinal differences may potentially be driven by a regression to the mean in controls, rather than being explicitly disease-related. Nevertheless, some regions do appear to show a consistent trajectory of change in early HD patients, while those of the controls 
remaining static (e.g. genu $F A$, right anterior limb of internal capsule $A D$, right superior corona radiata $A D$, left anterior corona radiata $R D)$.

Inferring specific biological processes underlying alterations in DTI metrics can be contentious [64]. Animal studies combining DTI and histology to model white matter damage $[65,66]$ and HD specifically [67] have indicated that differing pathological processes may underlie AD and RD measurements. Under this assumption, RD increases reflect demyelination [68], while changes in AD are more complex and time-dependent. Decreases in $A D$ are thought to occur in response to acute axonal damage and inflammation [69]; conversely $A D$ increases reflect expanded extra-cellular spaces resulting from Wallerian degeneration and other more gross atrophic processes $[70,71]$. However, we would caution against the over-interpretation of instances where, for example, AD was significant and RD was not. Cellular modifications caused by HD pathology undoubtedly affect white matter, but the mechanisms underlying this are unclear. Elements of intrinsic axonal degeneration, altered fibre coherence or configuration due to inflammation, decreased axonal density or demyelination all occur during the progression of HD and current DTI methods lack such aetiological specificity. More recent developments in diffusion MRI [72-76], may be more aetiologicallyinformative regarding microstructural white matter changes occurring during HD progression.

Analysis of clinical associations found a relationship between DBS and change in RD in the body of corpus callosum, with greater disease burden at baseline related to greater increases in RD over 15 months during the early stages of manifest HD. Again, it is the corpus callosum that is most significantly targeted and, notwithstanding the aforementioned caveats, this may reflect on-going demyelination in the corpus callosum of the more severe patients, in line with previous evidence for corpus callosum abnormalities in early HD $[18,19,24,27,63]$. The number of associations between DTI metrics and clinical measures that did not reach statistical significance was however, unexpectedly high. Poudel et al also found that when correlating FA with a series of clinical measures, only UHDRS-TMS scores were significant predictors of white matter microstructural 
changes [29], illustrating the potential difficulty in demonstrating clear relationships between clinical measures and longitudinal neuroimaging metrics. While this has been achieved using robust volumetric methods [36-38], issues regarding signal-to-noise ratio, reliability and validity hinder both DTI and many clinical measures and detecting associations may be better achieved by adopting novel diffusion-imaging techniques or by combining multiple imaging modalities with clinical data in a multivariate framework.

Combining data from multiple scanners can present limitations, however our previous work has demonstrated that the cross-site pooling of data does not introduce bias in DTI analyses [50]. Furthermore, longitudinal within-participant change measures were not confounded by scanner differences. Our study did not model any potentially confounding effects of medication on white matter metrics. However, given the considerable heterogeneity in medication usage among the participants, it would not have been possible to test medication effect systematically.

In conclusion, patients in the early stages of clinically-manifest HD show robust abnormalities in white matter structure throughout the brain, most robustly in the corpus callosum. Moreover, some of those regions appear to be undergoing microstructural degeneration as the disease progresses during the early diagnosed stages, with some evidence for a relationship between disease burden and structure of the corpus callosum. Despite these findings, our results demonstrate the difficulties in mapping effects of clinical variables onto a dynamically and heterogeneous pattern of brain changes that take place during early HD. Novel diffusion imaging methods which provide more information regarding the biological underpinnings of changes in white matter microstructure are required to further understand the heterogeneity of such alterations within HD. Furthermore, the use of multivariate modelling, which incorporates both imaging and clinical data, will also help to understand how white matter changes are related to clinical symptoms.

\section{Acknowledgements}


The authors would like to thank Shiva Keihaninejad for help with the tensor based registration methods. The authors would also like to thank the patients and controls who took part in this study and acknowledge the efforts of all the PADDINGTON investigators in collecting the data used in the study. This work has been supported by the European Union - PADDINGTON project and all authors, with the exception of RS, SG and $\mathrm{HZ}$ receive funding from this project. RS, SG and $\mathrm{HZ}$ are supported by the $\mathrm{CHDI} / \mathrm{High}$ Q Foundation, a not-for-profit organization dedicated to finding treatments for Huntington's disease. This work was undertaken at UCLH/UCL supported by the National Institute for Health Research [NIHR] University College London Hospitals [UCLH] Biomedical Research Centre [BRC].

\section{Conflict of interest}

The authors have no conflict of interest to report. 


\section{References}

[1] The Huntington's Disease Collaborative Research Group. A novel gene containing a trinucleotide repeat that is expanded and unstable on Huntington's disease chromosomes. The Huntington's Disease Collaborative Research Group. Cell. 1993;72(6):971-83.

[2] Rosas HD, Koroshetz WJ, Chen YI, Skeuse C, Vangel M, Cudkowicz ME, et al. Evidence for more widespread cerebral pathology in early HD: An MRI-based morphometric analysis. Neurology. 2003;60(10):1615-20.

[3] Tabrizi SJ, Langbehn DR, Leavitt BR, Roos RA, Durr A, Craufurd D, et al. Biological and clinical manifestations of Huntington's disease in the longitudinal TRACK-HD study: cross-sectional analysis of baseline data. Lancet neurology. 2009;8(9):791-801.

[4] Aylward EH, Codori AM, Rosenblatt A, Sherr M, Brandt J, Stine OC, et al. Rate of caudate atrophy in presymptomatic and symptomatic stages of Huntington's disease. Movement disorders : official journal of the Movement Disorder Society. 2000;15(3):552-60.

[5] Georgiou-Karistianis N, Gray MA, Dominguez DJ, Dymowski AR, Bohanna I, Johnston LA, et al. Automated differentiation of pre-diagnosis Huntington's disease from healthy control individuals based on quadratic discriminant analysis of the basal ganglia: the IMAGE-HD study. Neurobiology of disease. 2013;51:82-92.

[6] Georgiou-Karistianis N, Scahill R, Tabrizi SJ, Squitieri F, Aylward E. Structural MRI in Huntington's disease and recommendations for its potential use in clinical trials. Neuroscience and biobehavioral reviews. 2013;37(3):480-90.

[7] Bohanna I, Georgiou-Karistianis N, Sritharan A, Asadi H, Johnston L, Churchyard A, et al. Diffusion Tensor Imaging in Huntington's disease reveals distinct patterns of white matter degeneration associated with motor and cognitive deficits. Brain Imaging and Behavior. 2011;5(3):171-80. 
[8] Della Nave R, Ginestroni A, Tessa C, Giannelli M, Piacentini S, Filippi M, et al. Regional distribution and clinical correlates of white matter structural damage in Huntington disease: A tractbased spatial statistics study. American Journal of Neuroradiology. 2010;31(9):1675-81.

[9] Delmaire C, Dumas EM, Sharman MA, van den Bogaard SJ, Valabregue R, Jauffret C, et al. The structural correlates of functional deficits in early huntington's disease. Hum Brain Mapp. 2013;34(9):2141-53.

[10] Douaud G, Behrens TE, Poupon C, Cointepas Y, Jbabdi S, Gaura V, et al. In vivo evidence for the selective subcortical degeneration in Huntington's disease. Neurolmage. 2009;46(4):958-66. [11] Hobbs NZ, Cole JH, Farmer RE, Rees EM, Crawford HE, Malone IB, et al. Evaluation of multimodal, multi-site neuroimaging measures in Huntington's disease: Baseline results from the PADDINGTON study. Neurolmage: Clinical. 2013;2(1):204-11.

[12] Klöppel S, Draganski B, Golding CV, Chu C, Nagy Z, Cook PA, et al. White matter connections reflect changes in voluntary-guided saccades in pre-symptomatic Huntington's disease. Brain. 2008;131(1):196-204.

[13] Magnotta VA, Kim J, Koscik T, Beglinger LJ, Espinso D, Langbehn D, et al. Diffusion Tensor Imaging in Preclinical Huntington's Disease. Brain imaging and behavior. 2009;3(1):77-84.

[14] Matsui JT, Vaidya JG, Johnson HJ, Magnotta VA, Long JD, Mills JA, et al. Diffusion weighted imaging of prefrontal cortex in prodromal huntington's disease. Human brain mapping. 2014;35(4):1562-73.

[15] Reading SAJ, Yassa MA, Bakker A, Dziorny AC, Gourley LM, Yallapragada V, et al. Regional white matter change in pre-symptomatic Huntington's disease: A diffusion tensor imaging study. Psychiatry Research - Neuroimaging. 2005;140(1):55-62.

[16] Rizk-Jackson A, Stoffers D, Sheldon S, Kuperman J, Dale A, Goldstein J, et al. Evaluating imaging biomarkers for neurodegeneration in pre-symptomatic Huntington's disease using machine learning techniques. Neurolmage. 2011;56(2):788-96. 
[17] Stoffers D, Sheldon S, Kuperman JM, Goldstein J, Corey-Bloom J, Aron AR. Contrasting gray and white matter changes in preclinical Huntington disease: An MRI study. Neurology. 2010;74(15):1208-16.

[18] Di Paola M, Luders E, Cherubini A, Sanchez-Castaneda C, Thompson PM, Toga AW, et al. Multimodal MRI Analysis of the Corpus Callosum Reveals White Matter Differences in Presymptomatic and Early Huntington's Disease. Cereb Cort. 2012;10.1093/cercor/bhr360.

[19] Di Paola M, Phillips OR, Sanchez-Castaneda C, Di Pardo A, Maglione V, Caltagirone C, et al. MRI measures of corpus callosum iron and myelin in early Huntington's disease. Human brain mapping. 2013.

[20] Dumas EM, van den Bogaard SJ, Ruber ME, Reilman RR, Stout JC, Craufurd D, et al. Early changes in white matter pathways of the sensorimotor cortex in premanifest Huntington's disease. Hum Brain Mapp. 2012;33(1):203-12.

[21] Mascalchi M, Lolli F, Della Nave R, Tessa C, Petralli R, Gavazzi C, et al. Huntington disease: volumetric, diffusion-weighted, and magnetization transfer MR imaging of brain. Radiology. 2004;232(3):867-73.

[22] Muller HP, Glauche V, Novak MJ, Nguyen-Thanh T, Unrath A, Lahiri N, et al. Stability of white matter changes related to Huntington's disease in the presence of imaging noise: a DTI study. PLoS currents. 2011;3:RRN1232.

[23] Novak MJU, Seunarine KK, Gibbard CR, Hobbs NZ, Scahill RI, Clark CA, et al. White matter integrity in premanifest and early Huntington's disease is related to caudate loss and disease progression. Cortex; a journal devoted to the study of the nervous system and behavior. 2014;52(1):98-112.

[24] Phillips O, Sanchez-Castaneda C, Elifani F, Maglione V, Di Pardo A, Caltagirone C, et al. Tractography of the Corpus Callosum in Huntington's Disease. PLoS One. 2013;8(9).

[25] Phillips O, Squitieri F, Sanchez-Castaneda C, Elifani F, Caltagirone C, Sabatini U, et al. Deep white matter in Huntington's disease. PloS one. 2014;9(10). 
[26] Poudel GR, Stout JC, Domínguez D JF, Salmon L, Churchyard A, Chua P, et al. White matter connectivity reflects clinical and cognitive status in Huntington's disease. Neurobiology of disease. 2014;65:180-7.

[27] Rosas HD, Lee SY, Bender AC, Zaleta AK, Vangel M, Yu P, et al. Altered white matter microstructure in the corpus callosum in Huntington's disease: Implications for cortical "disconnection". Neurolmage. 2010;49(4):2995-3004.

[28] Rosas HD, Tuch DS, Hevelone ND, Zaleta AK, Vangel M, Hersch SM, et al. Diffusion tensor imaging in presymptomatic and early Huntington's disease: Selective white matter pathology and its relationship to clinical measures. Movement Disorders. 2006;21(9):1317-25.

[29] Poudel GR, Stout JC, Dominguez DJ, Churchyard A, Chua P, Egan GF, et al. Longitudinal change in white matter microstructure in Huntington's disease: The IMAGE-HD study. Neurobiology of disease. 2015;74:406-12.

[30] Odish OF, Leemans A, Reijntjes RH, van den Bogaard SJ, Dumas EM, Wolterbeek R, et al. Microstructural brain abnormalities in Huntington's disease: A two-year follow-up. Human brain mapping. 2015;36(6):2061-74.

[31] Aylward EH, Nopoulos PC, Ross CA, Langbehn DR, Pierson RK, Mills JA, et al. Longitudinal change in regional brain volumes in prodromal Huntington disease. Journal of Neurology, Neurosurgery and Psychiatry. 2011;82(4):405-10.

[32] Ciarmiello A, Cannella M, Lastoria S, Simonelli M, Frati L, Rubinsztein DC, et al. Brain whitematter volume loss and glucose hypometabolism precede the clinical symptoms of Huntington's disease. Journal of Nuclear Medicine. 2006;47(2):215-22.

[33] Hobbs NZ, Henley SM, Ridgway GR, Wild EJ, Barker RA, Scahill RI, et al. The progression of regional atrophy in premanifest and early Huntington's disease: a longitudinal voxel-based morphometry study. J Neurol, Neurosurg \& Psych. 2010;81(7):756-63. 
[34] Rosas HD, Reuter M, Doros G, Lee SY, Triggs T, Malarick K, et al. A tale of two factors: What determines the rate of progression in Huntington's disease? A longitudinal MRI study. Movement Disorders. 2011;26(9):1691-7.

[35] Squitieri F, Cannella M, Simonelli M, Sassone J, Martino T, Venditti E, et al. Distinct brain volume changes correlating with clinical stage, disease progression rate, mutation size, and age at onset prediction as early biomarkers of brain atrophy in Huntington's disease. CNS neuroscience \& therapeutics. 2009;15(1):1-11.

[36] Tabrizi SJ, Reilmann R, Roos RA, Durr A, Leavitt B, Owen G, et al. Potential endpoints for clinical trials in premanifest and early Huntington's disease in the TRACK-HD study: analysis of 24 month observational data. Lancet neurology. 2012;11(1):42-53.

[37] Tabrizi SJ, Scahill RI, Durr A, Roos RA, Leavitt BR, Jones R, et al. Biological and clinical changes in premanifest and early stage Huntington's disease in the TRACK-HD study: the 12-month longitudinal analysis. Lancet neurology. 2011;10(1):31-42.

[38] Tabrizi SJ, Scahill RI, Owen G, Durr A, Leavitt BR, Roos RA, et al. Predictors of phenotypic progression and disease onset in premanifest and early-stage Huntington's disease in the TRACK-HD study: analysis of 36-month observational data. The Lancet Neurology. 2013.

[39] Hobbs NZ, Farmer RE, Rees EM, Cole JH, Haider S, Malone IB, et al. Short-interval observational data to inform clinical trial design in Huntington's disease. Journal of neurology, neurosurgery, and psychiatry. 2015;10.1136/jnnp-2014-309768.

[40] Beaulieu C. The basis of anisotropic water diffusion in the nervous system - A technical review. NMR Biomed. 2002;15(7-8):435-55.

[41] Rees EM, Scahill RI, Hobbs NZ. Longitudinal Neuroimaging Biomarkers in Huntington's Disease. Journal of Huntington's Disease. 2013;2(1):21-39.

[42] Domínguez DJF, Egan GF, Gray MA, Poudel GR, Churchyard A, Chua P, et al. Multi-Modal Neuroimaging in Premanifest and Early Huntington's Disease: 18 Month Longitudinal Data from the IMAGE-HD Study. PloS one. 2013;8(9). 
[43] Vandenberghe W, Demaerel P, Dom R, Maes F. Diffusion-weighted versus volumetric imaging of the striatum in early symptomatic Huntington disease. Journal of neurology. 2009;256(1):109-14.

[44] Smith SM, Jenkinson M, Johansen-Berg H, Rueckert D, Nichols TE, Mackay CE, et al. Tractbased spatial statistics: Voxelwise analysis of multi-subject diffusion data. Neurolmage. 2006;31(4):1487-505.

[45] Weaver KE, Richards TL, Liang O, Laurino MY, Samii A, Aylward EH. Longitudinal diffusion tensor imaging in Huntington's Disease. Experimental neurology. 2009;216(2):525-9.

[46] Sritharan A, Egan GF, Johnston L, Horne M, Bradshaw JL, Bohanna I, et al. A longitudinal diffusion tensor imaging study in symptomatic Huntington's disease. Journal of Neurology, Neurosurgery and Psychiatry. 2010;81(3):257-62.

[47] Unified Huntington's Disease Rating Scale: reliability and consistency. Huntington Study Group. Movement disorders : official journal of the Movement Disorder Society. 1996;11(2):136-42. [48] Odish OF, Caeyenberghs K, Hosseini H, van den Bogaard SJ, Roos RA, Leemans A. Dynamics of the connectome in Huntington's disease: A longitudinal diffusion MRI study. Neurolmage Clinical. 2015;9:32-43.

[49] Shoulson I, Fahn S. Huntington disease: clinical care and evaluation. Neurology. $1979 ; 29(1): 1-3$.

[50] Müller HP, Grön G, Sprengelmeyer R, Kassubek J, Ludolph AC, Hobbs N, et al. Evaluating multicenter DTI data in Huntington's disease on site specific effects: An ex post facto approach. Neurolmage: Clinical. 2013;2(1):161-7.

[51] Cook PA, Bai Y, Nedjati-Gilani S, Seunarine KK, Hall MG, Parker GJ, et al., editors. Camino: Open-Source Diffusion-MRI Reconstruction and Processing. 14th Scientific Meeting of the International Society for Magnetic Resonance in Medicine; 2006; Seattle, WA, USA.

[52] Zhang H, Yushkevich PA, Alexander DC, Gee JC. Deformable registration of diffusion tensor MR images with explicit orientation optimization. Medical image analysis. 2006;10(5):764-85. 
[53] Zhang $\mathrm{H}$, Avants BB, Yushkevich PA, Woo JH, Wang S, McCluskey LF, et al. High-dimensional spatial normalization of diffusion tensor images improves the detection of white matter differences: An example study using amyotrophic lateral sclerosis. IEEE Transactions on Medical Imaging. 2007;26(11):1585-97.

[54] Wang Y, Gupta A, Liu Z, Zhang H, Escolar ML, Gilmore JH, et al. DTI registration in atlas based fiber analysis of infantile Krabbe disease. Neurolmage. 2011;55(4):1577-86.

[55] Muller MJ, Mazanek M, Weibrich C, Dellani PR, Stoeter P, Fellgiebel A. Distribution characteristics, reproducibility, and precision of region of interest-based hippocampal diffusion tensor imaging measures. AJNR American journal of neuroradiology. 2006;27(2):440-6.

[56] Keihaninejad S, Zhang H, Ryan N, Malone I, Frost C, Cardoso M, et al. Longitudinal changes in fiber tract integrity in Alzheimer's disease: A serial diffusion tensor imaging study. Alzheimer's \& Dementia. 2012;8(S4):30-1.

[57] Lee CE, Danielian LE, Thomasson D, Baker EH. Normal regional fractional anisotropy and apparent diffusion coefficient of the brain measured on a 3 T MR scanner. Neuroradiology. 2009;51(1):3-9.

[58] Mori S, Oishi K, Jiang H, Jiang L, Li X, Akhter K, et al. Stereotaxic white matter atlas based on diffusion tensor imaging in an ICBM template. Neurolmage. 2008;40(2):570-82.

[59] Cole JH, Farmer RE, Rees EM, Johnson HJ, Frost C, Scahill RI, et al. Test-Retest Reliability of Diffusion Tensor Imaging in Huntington's Disease. PLoS Curr. 2014;6.

[60] Ourselin S, Roche A, Subsol G, Pennec X, Ayache N. Reconstructing a 3D structure from serial histological sections. Image and Vision Computing. 2001;19(1-2):25-31.

[61] Modat M, Ridgway GR, Taylor ZA, Lehmann M, Barnes J, Hawkes DJ, et al. Fast free-form deformation using graphics processing units. Comput Methods Programs Biomed. 2010;98(3):27884.

[62] Benjamini Y, Hochberg Y. Controlling the False Discovery Rate - a Practical and Powerful Approach to Multiple Testing. J Roy Stat Soc B Met. 1995;57(1):289-300. 
[63] Crawford HE, Hobbs NZ, Keogh R, Langbehn DR, Frost C, Johnson H, et al. Corpus callosal atrophy in premanifest and early Huntington's disease. J Huntingtons Dis. 2013;2(4):517-26.

[64] Jones DK, Knosche TR, Turner R. White matter integrity, fiber count, and other fallacies: the do's and don'ts of diffusion MRI. Neurolmage. 2013;73:239-54.

[65] Song SK, Sun SW, Ju WK, Lin SJ, Cross AH, Neufeld AH. Diffusion tensor imaging detects and differentiates axon and myelin degeneration in mouse optic nerve after retinal ischemia. Neurolmage. 2003;20(3):1714-22.

[66] Sun SW, Liang HF, Cross AH, Song SK. Evolving Wallerian degeneration after transient retinal ischemia in mice characterized by diffusion tensor imaging. Neurolmage. 2008;40(1):1-10.

[67] Van Camp N, Blockx I, Camón L, De Vera N, Verhoye M, Veraart J, et al. A complementary diffusion tensor imaging (DTI)-histological study in a model of Huntington's disease. Neurobiology of Aging. 2012;33(5):945-59.

[68] Song SK, Yoshino J, Le TQ, Lin SJ, Sun SW, Cross AH, et al. Demyelination increases radial diffusivity in corpus callosum of mouse brain. Neurolmage. 2005;26(1):132-40.

[69] Budde MD, Xie M, Cross AH, Song SK. Axial Diffusivity Is the Primary Correlate of Axonal Injury in the Experimental Autoimmune Encephalomyelitis Spinal Cord: A Quantitative Pixelwise Analysis. J Neurosci. 2009;29(9):2805-13.

[70] Concha L, Gross DW, Wheatley BM, Beaulieu C. Diffusion tensor imaging of time-dependent axonal and myelin degradation after corpus callosotomy in epilepsy patients. Neurolmage. 2006;32(3):1090-9.

[71] Della Nave R, Ginestroni A, Diciotti S, Salvatore E, Soricelli A, Mascalchi M. Axial diffusivity is increased in the degenerating superior cerebellar peduncles of Friedreich's ataxia. Neuroradiology. 2011;53(5):367-72.

[72] Jensen JH, Helpern JA. MRI quantification of non-Gaussian water diffusion by kurtosis analysis. NMR Biomed. 2010;23(7):698-710. 
[73] Zhang H, Schneider T, Wheeler-Kingshott CA, Alexander DC. NODDI: Practical in vivo neurite orientation dispersion and density imaging of the human brain. Neurolmage. 2012;61(4):1000-16.

[74] Zhang H, Hubbard PL, Parker GJM, Alexander DC. Axon diameter mapping in the presence of orientation dispersion with diffusion MRI. Neurolmage. 2011;56(3):1301-15.

[75] Assaf Y, Blumenfeld-Katzir T, Yovel Y, Basser PJ. AxCaliber: A method for measuring axon diameter distribution from diffusion MRI. Magnetic Resonance in Medicine. 2008;59(6):1347-54. [76] Tournier JD, Mori S, Leemans A. Diffusion tensor imaging and beyond. Magnetic resonance in medicine : official journal of the Society of Magnetic Resonance in Medicine / Society of Magnetic Resonance in Medicine. 2011;65(6):1532-56.

[77] Penney JB, Vonsattel JP, MacDonald ME, Gusella JF, Myers RH. CAG repeat number governs the development rate of pathology in huntington's disease. Annals of Neurology. 1997;41(5):689-92. [78] Papadakis NG, Xing D, Huang CL, Hall LD, Carpenter TA. A comparative study of acquisition schemes for diffusion tensor imaging using MRI. Journal of magnetic resonance. 1999;137(1):67-82. [79] Jones DK. The effect of gradient sampling schemes on measures derived from diffusion tensor MRI: a Monte Carlo study. Magnetic resonance in medicine : official journal of the Society of Magnetic Resonance in Medicine / Society of Magnetic Resonance in Medicine. 2004;51(4):807-15. [80] Poonawalla AH, Zhou XJ. Analytical error propagation in diffusion anisotropy calculations. Journal of magnetic resonance imaging : JMRI. 2004;19(4):489-98.

[81] Barrio-Arranz G, de Luis-Garcia R, Tristan-Vega A, Martin-Fernandez M, Aja-Fernandez S. Impact of MR Acquisition Parameters on DTI Scalar Indexes: A Tractography Based Approach. PloS one. 2015;10(10):e0137905.

[82] Muller HP, Sussmuth SD, Landwehrmeyer GB, Ludolph A, Tabrizi SJ, Kloppel S, et al. Stability effects on results of diffusion tensor imaging analysis by reduction of the number of gradient directions due to motion artifacts: an application to presymptomatic Huntington's disease. PLoS Curr. 2011;3:RRN1292. 


\section{Tables}

Table 1. HD patient and control characteristics

\begin{tabular}{|c|c|c|c|c|}
\hline \multirow{2}{*}{$\begin{array}{l}\text { Baseline characteristics } \\
\mathbf{N}\end{array}$} & \multicolumn{2}{|c|}{ Controls } & \multicolumn{2}{|c|}{ Early HD patients } \\
\hline & 36 & & 48 & \\
\hline Age (Years) [Mean (SD) Range] & $51.82(8.75)$ & $28.98-66.64$ & $48.94(9.38)$ & $26.77-67.29$ \\
\hline Sex (Female/Male N (\%)) & $20 / 16$ & $(55.6 \%) /(44.4 \%)$ & $29 / 19$ & $(60.4 \%) /(39.6 \%)$ \\
\hline Education Level (ISCED) [Mean (SD) & $3.97(1.23)$ & $2-6$ & $3.42(1.27)$ & $2-6$ \\
\hline \multicolumn{5}{|l|}{ Range] } \\
\hline Baseline-15 month interval (Days) & $439.75(32.27)$ & $342-491$ & $452.52(43.44)$ & $370-565$ \\
\hline \multicolumn{5}{|l|}{ [Mean (SD) Range] } \\
\hline \multicolumn{5}{|l|}{ Centre } \\
\hline Leiden $\mathbf{N}(\%)$ & 9 & $(25 \%)$ & 11 & $(22.92 \%)$ \\
\hline London N (\%) & 10 & $(27.78 \%)$ & 13 & $(27.08 \%)$ \\
\hline Paris N (\%) & 8 & $(22.22 \%)$ & 9 & $(18.75 \%)$ \\
\hline Ulm N (\%) & 9 & $(25 \%)$ & 15 & $(31.25 \%)$ \\
\hline CAG [Mean (SD) Range] & & & $43.31(2.61)$ & $39-54$ \\
\hline Disease Burden Score [Mean (SD) & & & $366.59(85.66)$ & $226.41-559.18$ \\
\hline \multicolumn{5}{|l|}{ Range] } \\
\hline Total Functional Capacity [Mean (SD) & $12.97(0.17)$ & $12-13$ & $12.02(0.93)$ & $9-13$ \\
\hline \multicolumn{5}{|l|}{ Range] } \\
\hline Total Motor Score [Mean (SD) Range] & $1.44(1.89)$ & $0-7$ & $18.58(9.19)$ & $6-45$ \\
\hline $\begin{array}{l}\text { Disease burden score [77] calculated a } \\
\text { Classification of Education. CAG = tri-n }\end{array}$ & ex (cag-35.5) & $=$ standard devi & ISCED = Inte & nal Standard \\
\hline
\end{tabular}


Table 2. Baseline comparison of diffusion metrics in white matter regions between early HD patient and controls

\begin{tabular}{|c|c|c|c|c|c|c|}
\hline Brain region & Fractional Anisotropy & $P$ & Axial Diffusivity & $P$ & Radial Diffusivity & $P$ \\
\hline Cingulum (cingulate gyrus) - L & $-0.033[-0.055,-0.012]$ & 0.002 & $0.019[-0.009,0.047]$ & 0.188 & $0.055[0.025,0.085]$ & $<0.001$ \\
\hline Cingulum (cingulate gyrus) - $\mathbf{R}$ & $-0.031[-0.050,-0.011]$ & $0.002^{*}$ & $0.000[-0.026,0.027]$ & 0.975 & $0.042[0.015,0.069]$ & $0.002^{*}$ \\
\hline Corona radiata - Anterior - $\mathrm{L}$ & $-0.007[-0.019,0.005]$ & 0.264 & $0.037[0.019,0.055]$ & $<0.001 *$ & $0.025[0.007,0.044]$ & $0.006^{*}$ \\
\hline Corona radiata - Anterior - $\mathbf{R}$ & $-0.009[-0.024,0.006]$ & 0.245 & $0.039[0.017,0.060]$ & $<0.001 *$ & $0.027[0.004,0.050]$ & $0.023 *$ \\
\hline Corona radiata - Posterior - $\mathrm{L}$ & $-0.006[-0.021,0.008]$ & 0.383 & $0.037[0.018,0.056]$ & $<0.001 *$ & $0.024[0.007,0.042]$ & $0.007^{*}$ \\
\hline Corona radiata - Posterior - $\mathbf{R}$ & $-0.008[-0.023,0.006]$ & 0.273 & $0.038[0.016,0.061]$ & $0.001^{*}$ & $0.031[0.011,0.050]$ & $0.002^{*}$ \\
\hline Corona radiata - Superior - $\mathrm{L}$ & $-0.006[-0.019,0.007]$ & 0.349 & $0.040[0.022,0.059]$ & $<0.001 *$ & $0.024[0.010,0.038]$ & $0.001^{*}$ \\
\hline Corona radiata - Superior - $\mathbf{R}$ & $-0.006[-0.020,0.007]$ & 0.364 & $0.036[0.015,0.056]$ & $0.001^{*}$ & $0.021[0.007,0.035]$ & $0.003^{*}$ \\
\hline Corpus callosum - Body & $-0.023[-0.036,-0.010]$ & $<0.001 *$ & $0.060[0.038,0.081]$ & $<0.001 *$ & $0.054[0.032,0.076]$ & $<0.001^{*}$ \\
\hline Corpus callosum - Genu & $-0.026[-0.041,-0.011]$ & $0.001^{*}$ & $0.046[0.021,0.070]$ & $<0.001 *$ & $0.051[0.022,0.080]$ & $0.001 *$ \\
\hline Corpus callosum - Splenium & $-0.023[-0.034,-0.011]$ & $<0.001^{*}$ & $0.082[0.050,0.114]$ & $<0.001^{*}$ & $0.058[0.032,0.084]$ & $<0.001^{*}$ \\
\hline External capsule - $L$ & $-0.018[-0.028,-0.008]$ & $<0.001^{*}$ & $0.043[0.027,0.058]$ & $<0.001 *$ & $0.046[0.028,0.064]$ & $<0.001^{*}$ \\
\hline External capsule - $\mathbf{R}$ & $-0.011[-0.023,0.000]$ & 0.052 & $0.057[0.038,0.075]$ & $<0.001 *$ & $0.045[0.024,0.066]$ & $<0.001^{*}$ \\
\hline Internal capsule - Anterior limb - L & $0.001[-0.012,0.014]$ & 0.927 & $0.064(0.046,0.083)$ & $<0.001 *$ & $0.031[0.014,0.047]$ & $<0.001 *$ \\
\hline Internal capsule - Anterior limb - R & $0.000[-0.012,0.013]$ & 0.948 & $0.060[0.040,0.080]$ & $<0.001^{*}$ & $0.039[0.021,0.057]$ & $<0.001^{*}$ \\
\hline Internal capsule - Retrolenticular - L & $0.003[-0.011,0.017]$ & 0.666 & $0.055[0.034,0.077]$ & $<0.001^{*}$ & $0.018[-0.001,0.037]$ & 0.059 \\
\hline Internal capsule - Retrolenticular - $\mathbf{R}$ & $-0.000[-0.014,0.014]$ & 0.981 & $0.047[0.028,0.067]$ & $<0.001^{*}$ & $0.020[0.002,0.037]$ & $0.025^{*}$ \\
\hline Pontine crossing tract & $-0.023[-0.041,-0.005]$ & $0.012^{*}$ & $0.019[-0.012,0.051]$ & 0.235 & $0.033[0.006,0.060]$ & $0.016 *$ \\
\hline Posterior thalamic radiation - $\mathrm{L}$ & $-0.000[-0.015,0.015]$ & 0.983 & $0.032[0.004,0.059]$ & $0.023^{*}$ & $0.016[-0.007,0.039]$ & 0.176 \\
\hline Posterior thalamic radiation $-\mathbf{R}$ & $-0.008[-0.025,0.009]$ & 0.366 & $0.035[0.002,0.067]$ & $0.035^{*}$ & $0.025[-0.005,0.055]$ & 0.106 \\
\hline Sagittal stratum - L & $-0.023[-0.036,-0.010]$ & $0.001^{*}$ & $0.059[0.028,0.090]$ & $<0.001 *$ & $0.062[0.037,0.088]$ & $<0.001^{*}$ \\
\hline Sagittal stratum - R & $-0.016[-0.031,-0.002]$ & 0.029 & $0.034[0.002,0.067]$ & $0.039^{*}$ & $0.035[0.008,0.061]$ & 0.010 \\
\hline Superior longitudinal fasciculus - L & $-0.011[-0.024,0.001]$ & 0.083 & $0.025[0.011,0.040]$ & $0.001^{*}$ & $0.027[0.010,0.044]$ & $0.002^{*}$ \\
\hline Superior longitudinal fasciculus - $\mathbf{R}$ & $-0.014[-0.026,-0.001]$ & 0.032 & $0.031[0.017,0.045]$ & $<0.001 *$ & $0.031[0.014,0.049]$ & $<0.001^{*}$ \\
\hline
\end{tabular}


Table 3. Early HD and control longitudinal rate of change comparison

\begin{tabular}{|c|c|c|c|c|c|c|}
\hline Brain Region & Fractional Anisotropy & $P$ & Axial Diffusivity & $P$ & Radial Diffusivity & $P$ \\
\hline Cingulum (cingulate gyrus) - L & $0.002[-0.006,0.010]$ & 0.627 & $-0.002[-0.013,0.008]$ & 0.693 & $-0.004[0.015,0.007]$ & 0.489 \\
\hline Cingulum (cingulate gyrus) - $\mathbf{R}$ & $-0.004[-0.010,0.002]$ & 0.216 & $0.003[-0.008,0.015]$ & 0.578 & $0.006[-0.003,0.017]$ & 0.208 \\
\hline Corona radiata - Anterior - $\mathrm{L}$ & $-0.005[-0.012,0.000]$ & 0.077 & $0.017[0.005,0.028]$ & $0.003 *$ & $0.015[0.004,0.026]$ & $0.006^{*}$ \\
\hline Corona radiata - Anterior - $\mathbf{R}$ & $-0.007[-0.014,-0.000]$ & 0.035 & $0.007[-0.004,0.018]$ & 0.216 & $0.011[0.000,0.021]$ & 0.042 \\
\hline Corona radiata - Posterior - L & $0.001[-0.004,0.008]$ & 0.568 & $0.002[-0.007,0.012]$ & 0.597 & $0.000[-0.008,0.010]$ & 0.856 \\
\hline Corona radiata - Posterior - $\mathbf{R}$ & $-0.002[-0.007,0.003]$ & 0.435 & $0.008[0.000,0.015]$ & 0.028 & $0.004[-0.002,0.011]$ & 0.196 \\
\hline Corona radiata - Superior - L & $-0.001[-0.005,0.003]$ & 0.615 & $0.008[0.001,0.015]$ & 0.020 & $0.004[-0.001,0.011]$ & 0.155 \\
\hline Corona radiata - Superior - $\mathbf{R}$ & $-0.002[-0.008,0.002]$ & 0.302 & $0.009[0.002,0.016]$ & $0.006^{*}$ & $0.005[-0.001,0.012]$ & 0.111 \\
\hline Corpus callosum - Body & $-0.012[-0.017,-0.007]$ & $<0.001 *$ & $0.012[0.001,0.022]$ & 0.020 & $0.020[0.011,0.028]$ & $<0.001^{*}$ \\
\hline Corpus callosum - Genu & $-0.006[-0.010,-0.002]$ & $0.004^{*}$ & $0.023[0.014,0.033]$ & $<0.001 *$ & $0.018[0.010,0.026]$ & $<0.001^{*}$ \\
\hline Corpus callosum - Splenium & $-0.000[-0.005,0.004]$ & 0.879 & $0.004[-0.007,0.016]$ & 0.441 & $0.001[-0.008,0.010]$ & 0.808 \\
\hline External capsule - L & $-0.004[-0.009,0.000]$ & 0.092 & $0.006[-0.003,0.015]$ & 0.193 & $0.007[0.000,0.015]$ & 0.044 \\
\hline External capsule - $\mathbf{R}$ & $-0.004[-0.010,0.001]$ & 0.137 & $0.008[-0.002,0.019]$ & 0.134 & $0.008[-0.001,0.018]$ & 0.090 \\
\hline Internal capsule - Anterior limb - L & $0.003[-0.003,0.010]$ & 0.288 & $0.011[0.000,0.022]$ & 0.041 & $0.000[-0.009,0.009]$ & 0.943 \\
\hline Internal capsule - Anterior limb - R & $-0.004[-0.012,0.004]$ & 0.365 & $0.026[0.012,0.041]$ & $<0.001^{*}$ & $0.016[0.003,0.030]$ & 0.012 \\
\hline Internal capsule - Retrolenticular - L & $0.003[-0.006,0.013]$ & 0.521 & $0.003[-0.009,0.016]$ & 0.579 & $-0.001[-0.014,0.012]$ & 0.834 \\
\hline Internal capsule - Retrolenticular - $R$ & $0.001[-0.006,0.009]$ & 0.695 & $0.001[-0.011,0.014]$ & 0.821 & $-0.001[-0.011,0.008]$ & 0.767 \\
\hline Pontine crossing tract & $0.018[0.002,0.034]$ & 0.025 & $-0.002[-0.027,0.023]$ & 0.875 & $-0.016[-0.040,0.007]$ & 0.170 \\
\hline Posterior thalamic radiation - $\mathrm{L}$ & $-0.005[-0.014,0.003]$ & 0.254 & $0.011[-0.005,0.028]$ & 0.186 & $0.011[-0.002,0.024]$ & 0.099 \\
\hline Posterior thalamic radiation - $\mathbf{R}$ & $0.000[-0.007,0.007]$ & 0.974 & $-0.001[-0.015,0.011]$ & 0.807 & $0.000[-0.010,0.011]$ & 0.924 \\
\hline Sagittal stratum - L & $0.003[-0.006,0.012]$ & 0.539 & $0.021[0.000,0.043]$ & 0.049 & $0.009[-0.007,0.025]$ & 0.281 \\
\hline Sagittal stratum - $\mathbf{R}$ & $-0.005[-0.012,0.001]$ & 0.114 & $0.006[-0.009,0.021]$ & 0.427 & $0.012[0.001,0.023]$ & 0.030 \\
\hline Superior longitudinal fasciculus - L & $-0.000[-0.005,0.003]$ & 0.708 & $0.002[-0.005,0.010]$ & 0.516 & $0.003[-0.003,0.009]$ & 0.361 \\
\hline Superior longitudinal fasciculus - $\mathbf{R}$ & $-0.002[-0.006,0.001]$ & 0.270 & $0.004[-0.002,0.010]$ & 0.214 & $0.002[-0.002,0.008]$ & 0.287 \\
\hline
\end{tabular}


Table 4. Association between 15-month change and CAG repeat length or baseline Disease Burden Score

\begin{tabular}{|c|c|c|c|c|}
\hline & \multicolumn{2}{|l|}{ CAG } & \multicolumn{2}{|c|}{ Disease Burden } \\
\hline & Estimate $[95 \% \mathrm{Cl}]^{+}$ & $P$ & Estimate $[95 \% \mathrm{Cl}]^{+}$ & $P$ \\
\hline Corpus callosum - Genu AD & $4.07[-4.58,12.73]$ & 0.35 & $0.08[-0.12,0.29]$ & 0.41 \\
\hline Corpus callosum - Genu FA & $-2.43[-6.08,1.22]$ & 0.19 & $-0.07[-0.15,0.02]$ & 0.11 \\
\hline Corpus callosum - Genu RD & $3.88[-3.84,11.6]$ & 0.32 & $0.11[-0.07,0.29]$ & 0.23 \\
\hline Corona radiata - Anterior - L AD & $0.92[-10.83,12.68]$ & 0.88 & $0.01[-0.27,0.28]$ & 0.97 \\
\hline Corona radiata - Anterior - L RD & $55.60[-475.32,586.57]$ & 0.83 & $-1.1[-13.47,11.27]$ & 0.86 \\
\hline Internal capsule - Anterior limb - R AD & $13.70[-0.41,27.87]$ & 0.06 & $0.37[0.05,0.69]$ & 0.03 \\
\hline Corona radiata - Superior-R AD & $5.42[0.06,10.79]$ & 0.05 & $0.14[0.01,0.26]$ & 0.03 \\
\hline Corpus callosum - Body FA & $-4.16[-8.07,-0.25]$ & 0.04 & $-0.10[-0.19,-0.01]$ & 0.04 \\
\hline Corpus callosum - Body RD & $8.70[2.02,15.39]$ & 0.01 & $0.23[0.07,0.38]$ & $<0.01^{*}$ \\
\hline $\begin{array}{l}\text { All analyses adjusted for age, sex and } s \\
+\times 10^{-6} \\
* \text { Statistically significant after FDR corr } \\
\text { FA = Fractional anisotropy } \\
\text { AD = Axial diffusivity } \\
\text { RD = Radial diffusivity } \\
L=\text { Left, } R=\text { Right }\end{array}$ & $\begin{array}{l}\text { Idy site. } \\
\text { tion }\end{array}$ & & & \\
\hline
\end{tabular}




\section{Figure legends}

\section{Figure 1.}

Overview of the unbiased longitudinal registration methods. (A) Within-subject registration performed on tensor images using an initial affine and subsequent non-linear step in DTI-TK to warp images from each visit to a withinsubject 'mid-space'. A within-subject average template was then generated. (B) These within-subject average images were registered together, again using DTI-TK, to define a longitudinal between-subject space. The initial input images were then warped to this between-subject space and averaged to form a longitudinal group template, specific to the study. (C) Using NiftyReg to calculate affine and non-linear registrations, the FA image from the ICBM-DTI-81 atlas was then warped to match the group template. The parameters of this registration were then applied to ICBM-DTI-81 labels. (D) Finally, subject and visit specific metrics for each ROI were calculated.

\section{Figure 2.}

The figure depicts the regions-of-interest (ROIs) that showed significant differences in rates of change in DTI metrics over 15 months, when comparing early HD patients and controls. The body (red) and genu (green) of the corpus callosum, along with the left anterior corona radiata (yellow) and right superior corona radiata (magenta) and anterior limb of internal capsule (blue) are shown. A) Coronal, B) sagittal and C) axial 2D slices, along with D) 3D representation are displayed in order to illustrate the spatial relatedness of the five significant regions.

\section{Figure 3.}

Longitudinal trajectories of DTI metrics for early HD patients and controls for all significant metric-ROI combinations. The fitted values depicted were calculated for hypothetical participants age 50, with a 50/50 male-female split and a $25 \%$ contribution from each site. Points are plotted at each assessment (baseline, 6 months, 15 months), with the dashed line representing early HD patients and the whole line representing controls. 
Figures

Figure 1.

(A)

Subject 1
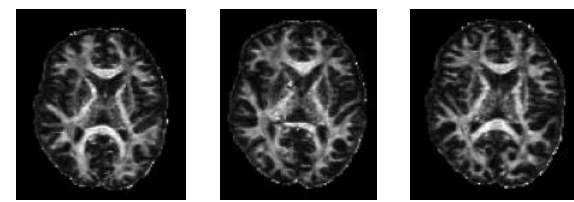

Subject $n$

Baseline

6 month

15 month
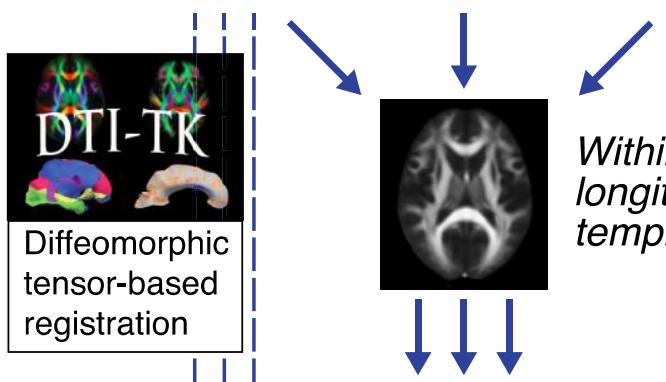

Within-subject longitudinal template

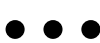

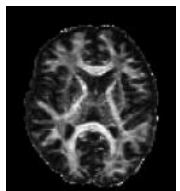

Baseline

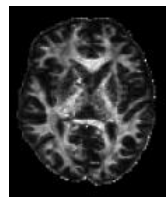

6 month

15 month
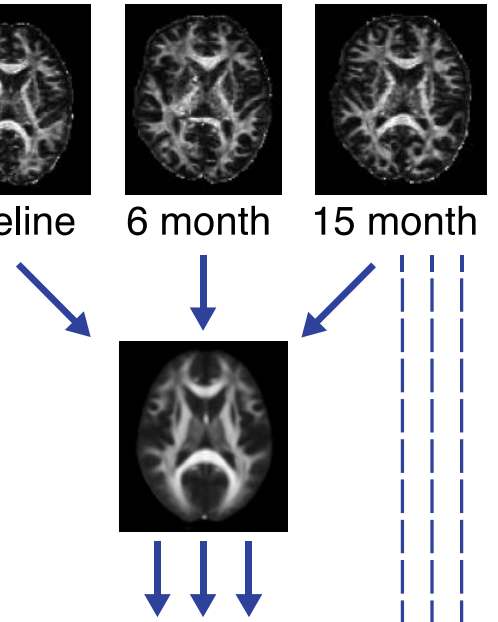

I I ।

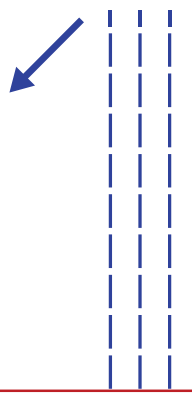

Within-subject $n$ : template space Baseline 6 month 15 month

(B)

Within-subject 1: template space Baseline 6 month 15 month
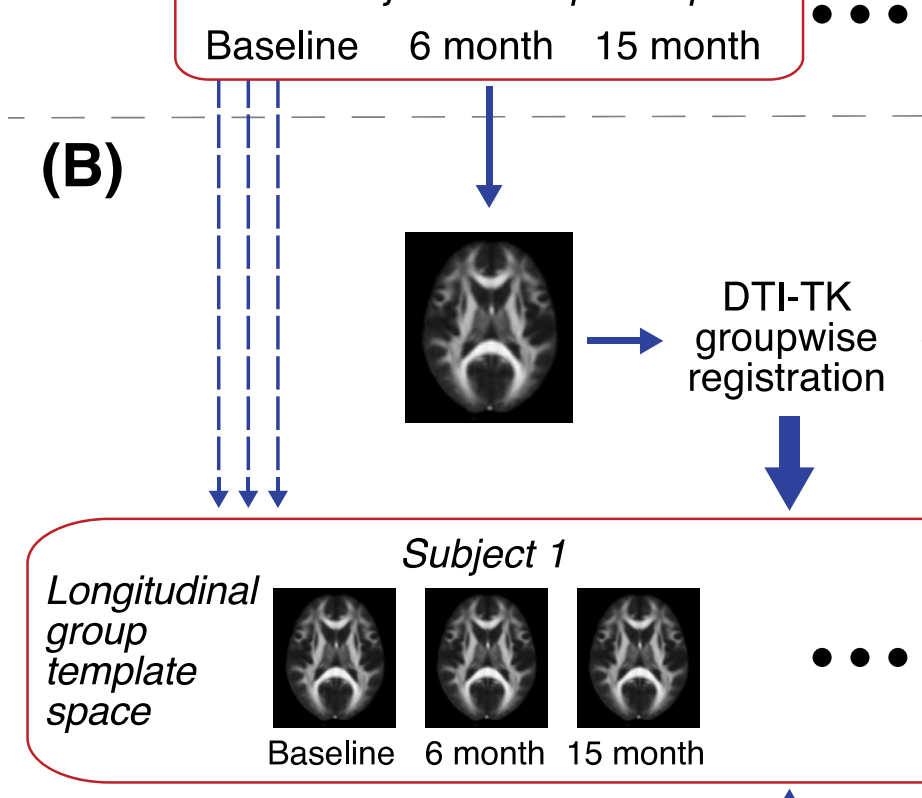

(C)

NiftyReg Affine + non-linear registration
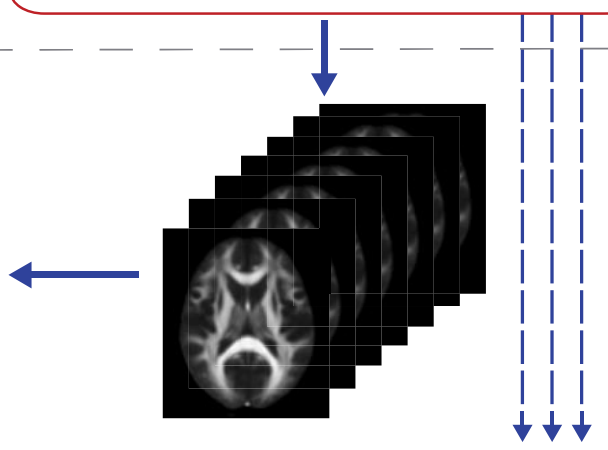

Longitudina group template space Subject $n$
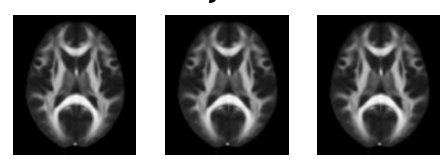

Baseline 6 month 15 month

ICBM-DTI-81 atlas

FA image \& ROI labels
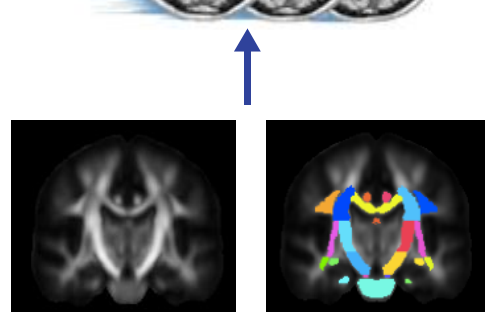

(D)

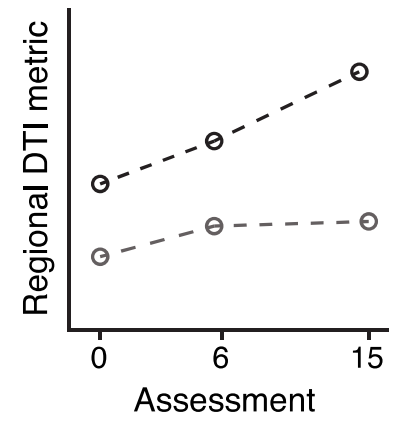


Figure 2.
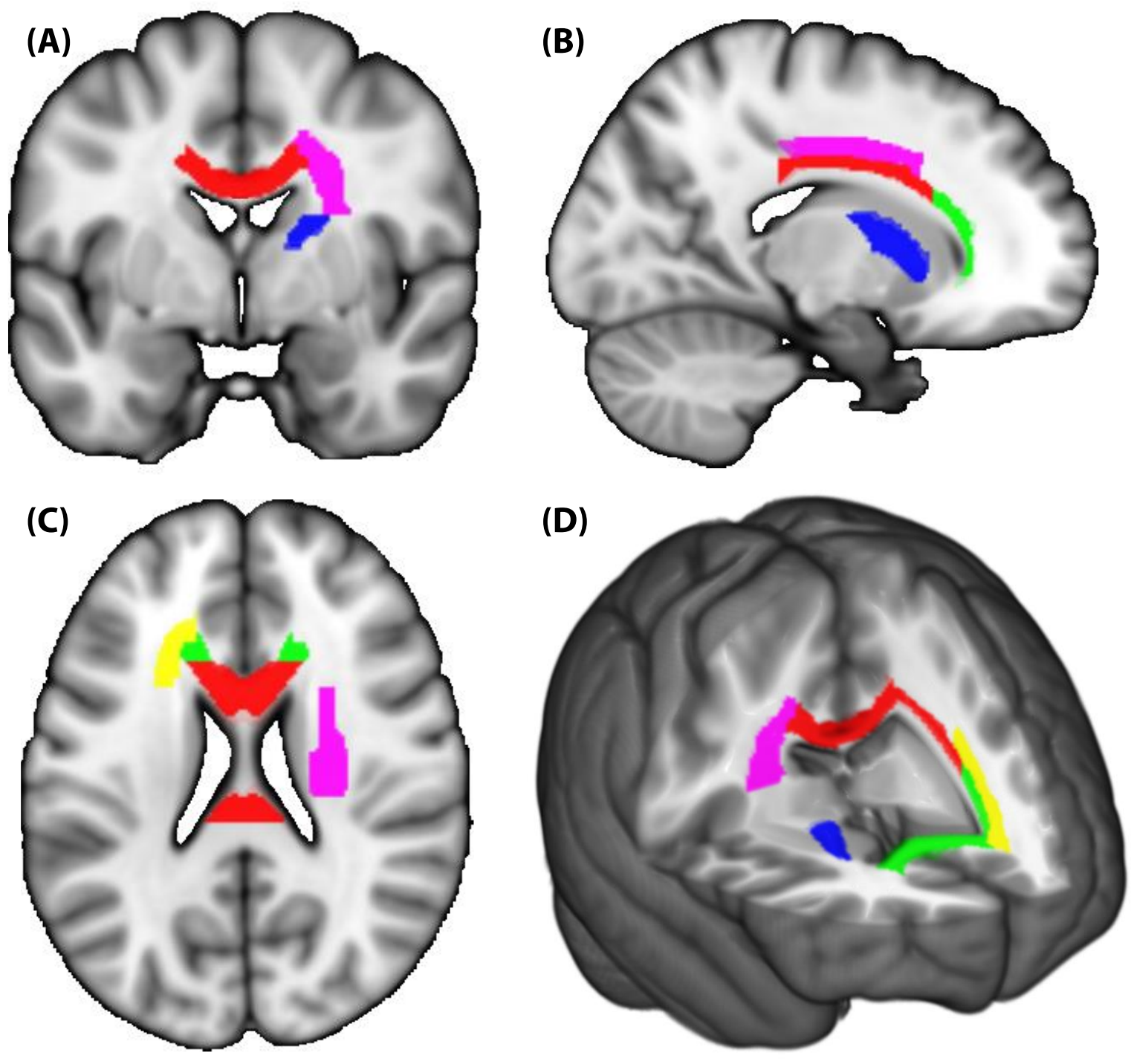
Figure 3.
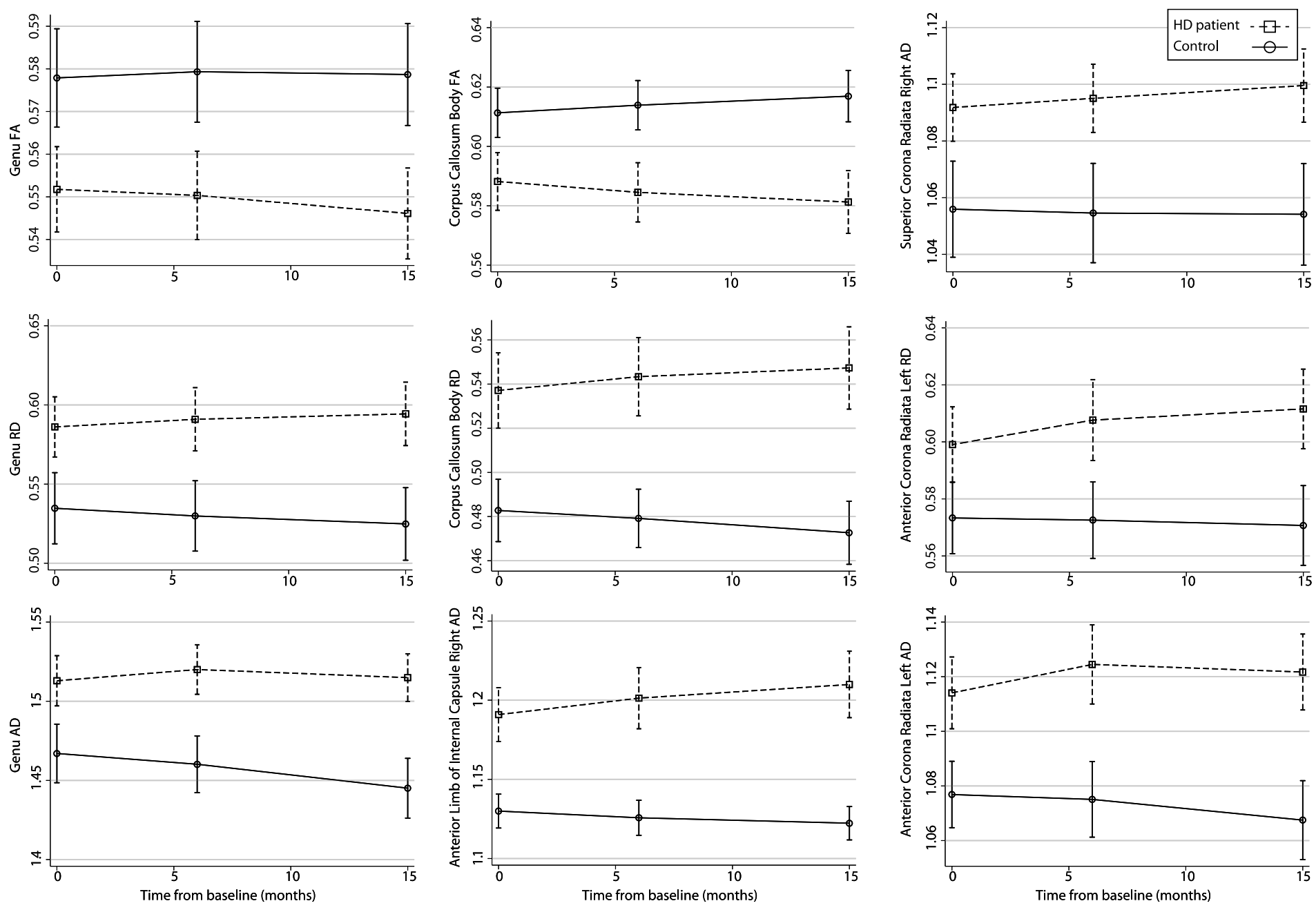


\section{Supplementary Information}

\section{Supplementary Methods}

\section{Diffusion Tensor Imaging: Gradient Directions}

We acquired DTI data in 42 gradient directions from three sites (London, Paris, Leiden) and in 47 gradient directions from one site (Ulm). The number of gradient directions is an important component within a DTI sequence and can impact the accuracy of tensor estimation and subsequent DTI metric values. However, while studies have shown that an increase in number of gradient directions from 6 to 12, for example, can have a substantial effect on the precision of DTI measurements [78] the optimal number of gradient directions has an upper limit. Jones has shown that 20 directions are required to achieve a robust estimate of anisotropy, while 30 directions are required for robust estimation of the tensor orientation and MD [79]. Similarly, Poonawalla et al. have shown that increasing the number of gradient directions has progressively less impact on diffusion metrics, with a plateau around 30-35 gradient directions [80]. Finally, it should be noted that error bias is related to SNR and remains unaffected by an increase in the number of gradient directions [81]. A recent multisite study in premanifest HD patients investigated the impact of increasing number of gradient directions in a dataset of premanifest Huntington's disease patients and controls on the quality of the FA maps. This study showed that there was still high agreement between FA maps that had included all gradient directions compared to those where up to 46 gradient directions were excluded [82].

\section{Diffusion Tensor Imaging: Motion}

Motion can be a major confounding factor in diffusion MRI. We, therefore, adopted a number of measures to combat the effects of motion on the quality of our DTI data. Firstly, during the scanning session, all participants had padding surrounding the head to reduce motion and were asked to keep very still while the DTI sequence was performed. We are aware, that participants with HD are more prone to movement within the scanner given the nature of their condition and therefore, may induce more motion artefacts than a healthy control group. It is important to note that, however, that, all but one of the patients were Stage 1-HD, having only recently received clinical motor diagnosis of HD and so their movement within the scanner would be potentially lower than other HD patients in more advanced stages of the disease. Nevertheless, it was likely that HD patient data would show more effect of motion and so we implemented a strict visual quality control procedure. For each participant, we inspected every slice of all gradient and non-gradient volumes for each timepoint. This is a very effective way of identifying motion-related artefacts, 
while also ensuring even sampling (which is not the case with automated quality control software). Following tensorfit and at every subsequent stage of pre-processing, the data were visually inspected for possible effects of motion. Most importantly, prior to any processing, we corrected the data for any motion-induced currents using eddy-current correction in FSL; the b-vectors (gradient directions) were then updated accordingly and this updated motion-related information used in subsequent processing steps. A recent study investigated the effects of movement on the quality of DTI data in the HD population, using an outlier detection algorithm to compare the index DWI direction against a weighted average computed from all other directions of the same subject [22]. This study showed that no significant differences were observed when independently comparing groups of patients, both with and without removal of DWI volumes that contained artefacts and that further still, hypothesized white matter differences based on the existing literature were still detectable.

\section{Correction for white matter atrophy}

We chose not to control for white matter atrophy, because we believe that the disease processes underlying macroscopic white matter volume changes are strongly related to those which result in white matter microstructural changes. Consequently, co-varying for white matter atrophy would confound the disease effect under investigation. 
SI Table 1. Comparison of excluded and included HD patient demographics

\begin{tabular}{lcccc}
\hline Baseline characteristics & \multicolumn{1}{c}{ Excluded HD patients } & Included HD patients \\
\hline $\mathrm{N}$ & 13 & & 48 & \\
Age (Years) [Mean (SD) Range] & $47.56(15.54)$ & $23.49-65.94$ & $48.94(9.38)$ & $26.77-67.29$ \\
Sex (Female/Male N (\%)) & $8 / 5$ & $(61.5 \%) /(38.5 \%)$ & $29 / 19$ & $(60.4 \%) /(39.6 \%)$ \\
Education Level (ISCED) [Mean (SD) Range] & $3.46(1.27)$ & $2-6$ & $3.42(1.27)$ & $2-6$ \\
Centre & & & & \\
$\quad$ Leiden N (\%) & 6 & $(46.15 \%)$ & 11 & $(22.92 \%)$ \\
$\quad$ London N (\%) & 3 & $(23.08 \%)$ & 13 & $(27.08 \%)$ \\
$\quad$ Paris N (\%) & 4 & $(30.77 \%)$ & 9 & $(18.75 \%)$ \\
$\quad$ Ulm N (\%) & 0 & $(0 \%)$ & 15 & $(31.25 \%)$ \\
CAG [Mean (SD) Range] & $45.46(4.54)$ & $39-53$ & $43.31(2.61)$ & $39-54$ \\
Disease Burden Score [Mean (SD) Range] & $412.87(75.46)$ & $230.77-511.07$ & $366.59(85.66)$ & $226.41-559.18$ \\
Total Functional Capacity [Mean (SD) Range] & $10.69(2.36)$ & $5-13$ & $12.02(0.93)$ & $9-13$ \\
Total Motor Score [Mean (SD) Range] & 25.54 & $6-58$ & $18.58(9.19)$ & $6-45$ \\
\hline Disease burden Score calculated as [age x (cag-35.5)] & & & & \\
SD = standard deviation & & & & \\
ISCED = International Standard Classification of Education & & & & \\
CAG = tri-nucleotide repeat length. & & & & \\
\hline
\end{tabular}


SI Table 2. Longitudinal site specific interaction

\begin{tabular}{|c|c|c|c|c|c|}
\hline \multirow[t]{3}{*}{ Region and DTI metric } & \multicolumn{4}{|c|}{ Site } & \multirow[t]{2}{*}{$P$ value for interaction } \\
\hline & Leiden & London & Paris & Ulm & \\
\hline & \multicolumn{4}{|c|}{ Baseline between-group differences } & \\
\hline Cingulum Cingulate - L - FA & $-0.042[-0.084,-0.001]^{*}$ & $0.011[-0.051,0.072]$ & $0.013[-0.046,0.072]$ & $0.013[-0.053,0.079]$ & \\
\hline Cingulum Cingulate - L - RD & $0.097[0.04,0.153]^{*}$ & $-0.062[-0.147,0.023]$ & $-0.059[-0.14,0.022]$ & $-0.057[-0.148,0.035]$ & \\
\hline Post thalamic radiation - L - RD & $-0.016[-0.06,0.029]$ & $0.04[-0.026,0.106]$ & $0.037[-0.026,0.1]$ & $0.058[-0.012,0.129]$ & \\
\hline \multirow[t]{2}{*}{ Sagittal Stratum - R - RD } & $0.054[0.003,0.105]^{*}$ & $-0.01[-0.086,0.065]$ & $-0.026[-0.098,0.047]$ & $-0.048[-0.129,0.033]$ & \\
\hline & \multicolumn{4}{|c|}{ Longitudinal between-group difference (15 month change) } & \\
\hline Cingulum Cingulate Left FA & $0.021[0.007,0.035]^{*}$ & $-0.014[-0.028,0.001]$ & $-0.009[-0.022,0.004]$ & $0.001[-0.015,0.018]$ & $0.001^{*}$ \\
\hline Cingulum Cingulate Left RD & $-0.032[-0.052,-0.012]^{*}$ & $0.018[-0.003,0.04]$ & $0.012[-0.007,0.031]$ & $-0.001[-0.025,0.022]$ & $0.001^{*}$ \\
\hline Post thalamic radiation Left RD & $0.046[0.023,0.069]^{*}$ & $-0.014[-0.04,0.011]$ & $0.006[-0.017,0.029]$ & $-0.005[-0.034,0.023]$ & $0.003^{*}$ \\
\hline Sagittal Stratum Right RD & $0.009[-0.011,0.029]$ & $-0.02[-0.041,0.002]$ & $0.028[0.008,0.047]^{*}$ & $0.03[0.006,0.054]^{*}$ & $0.006^{*}$ \\
\hline \multicolumn{6}{|c|}{$\begin{array}{l}\text { Site specific results from the primary analysis for } 4 \text { metrics suggesting site interactions were present after adjustment } \\
\text { diffusion metric. } \\
\text { Values are in the form of adjusted group difference estimates, with } 95 \% \text { confidence intervals presented in brackets. } \\
\text { * Denotes } p \text {-value }>0.05 \text { uncorrected. } \\
\text { FA = Fractional anisotropy } \\
\text { RD = Radial diffusivity } \\
L=\text { Left, } R=\text { Right }\end{array}$} \\
\hline
\end{tabular}

